\title{
Ni-W Amorphous/Nanocrystalline Duplex Composite Produced by Electrodeposition
}

\author{
Yoshihisa Kimoto $^{1}$, Akihito Giga ${ }^{1}$, Tadakatsu Ohkubo ${ }^{2}$, \\ Yorinobu Takigawa $^{1}$, K. Hono ${ }^{2}$ and Kenji Higashi ${ }^{1}$ \\ ${ }^{1}$ Department of Materials Science, Graduate School of Engineering, Osaka Prefecture University, \\ Sakai 599-8531, Japan \\ ${ }^{2}$ National Institute for Materials Science, Tsukuba 305-0047, Japan
}

\begin{abstract}
Detailed characterizations are performed to identify the nanostructures of electrodeposited Ni-W alloys with grain size of 5 and 8 nm. Three-dimensional atom probe (3DAP) analyses have clarified that, in both alloys, experimentally measured W-concentration distributions fit well to the bimodal binomial distribution compared to the single binomial distribution. This result indicates the coexistence of $\mathrm{W}$-depleted and W-enriched phases in the alloys. Nano-beam diffraction (NBD) patterns and energy dispersive x-ray spectroscopy (EDS) analyses revealed that the W-enriched phase is amorphous and that the W-depleted phase is nanocrystalline. The W-concentration visualizations on the cross section of 3DAP analysis volume identify them as amorphous/nanocrystalline duplex composites. [doi:10.2320/matertrans.48.996]
\end{abstract}

(Received November 24, 2006; Accepted March 2, 2007; Published April 25, 2007)

Keywords: nanocrystalline materials, three-dimensional atom probe, duplex composite, nickel alloys

\section{Introduction}

Nanocrystalline metals and alloys have inspired many researchers with great expectations of novel physical phenomena and unique material properties. They are roughly categorized into nanocrystalline metals, ${ }^{1)}$ metallic glasses ${ }^{2)}$ and metal nanocomposits. ${ }^{3)}$ A plenty of grain boundaries and interfaces in nanocrystalline materials are the essential trigger for the unique properties. The mechanical, magnetic and electrical properties of the nanocrystalline materials sometimes break the conventional scaling laws. For instance, many studies have reported that the hardness ${ }^{4}$ (or yield strength $^{5)}$ ) peaks out at the grain size of around a dozen nanometers before reaching amorphous limit, termed HallPetch breakdown. Similar trend is true for the coercivity of nanocrystalline ferromagnetic alloys. ${ }^{6}$ Such nonmonotonies not only attract the academic interests for elucidating the mechanism but also enable us to optimize the properties for industrial applications.

Generally, amorphous/nanocrystalline composites have been prepared through crystal nucleation and growth by the annealing of amorphous precursors. Electrodeposition techniques have also been used to produce a wide variety of nanostructured materials, including nanocrystalline metals and alloys, element metal/nanoparticle composites, alloy/ nanoparticle composites, nanomultilayers, and so forth. ${ }^{7)}$ Few studies ${ }^{8-10)}$ have reported the electrodeposited amorphous/nanocrystalline alloy composites. In electrodeposited $\mathrm{Ni}-\mathrm{W}$ alloys, it has been generally recognized that the structure changes from the single nanocrystalline (fcc Ni solid solution) phase to single amorphous phase at the $\mathrm{W}$ concentration of around 20 at\%. ${ }^{11,12)}$

The conceptual model of grain-boundary phase has been employed to describe the dependence of the mechanical property on the grain size. The composite model ${ }^{13,14)}$ explains that Hall-Petch breakdown is attributed to the increased volume fraction of a comparably soft grain boundary phase as the grain size decreases. Somekawa, et al. suggested that nanocrystalline and amorphous phases coexists in Ni-W alloys with grain size of $5 \mathrm{~nm}$, and the amorphous phase affect the deformation and fracture. ${ }^{15)}$ However, since the nanostructured metals can be composed of nanocrystals, nano-precipitates and nano-clusters, and/or amorphous phase, high-resolution analysis techniques are vitally necessary to observe and identify the nanostructures.

A three-dimensional atom probe (3DAP) is a powerful tool to investigate such nanostructures both qualitatively and quantitatively. ${ }^{16-18)}$ 3DAP data involves the element and three-dimensional coordinates of the atom in the analysis volumes of an order of $10 \times 10 \times 100 \mathrm{~nm}^{3}$, and the atomic distribution can be directly visualized. Choi, et al. have carried out the 3DAP analyses of electrodeposited Ni-18 at $\%$ $\mathrm{W}$ alloys before and after aging, and concluded that the thermal stability of the alloys is mainly due to the extremely low mobility of W atoms. ${ }^{19)}$ Detor et al. have analyzed the spatial fluctuations of the $\mathrm{W}$ concentration in electrodeposited Ni-W alloys, and compared the data with the McLean segregation isotherm to quantify the segregation energy. ${ }^{20)}$

In the present study, the nano-phases in these electrodeposited Ni-W alloys with grain sizes of 5 and $8 \mathrm{~nm}$ have been characterized by 3DAP and the nano beam diffraction (NBD) technique. The statistical analysis of the 3DAP data have revealed that the electrodeposited Ni-W alloys, which have been recognized as single nanocrystalline alloys, are proved to be in fact duplex composites of $\mathrm{W}$-depleted and $\mathrm{W}$ enriched phases. Each phase in the duplex composites was identified from by NBD patterns. The volume fractions of the two phases have been quantified using the 3DAP data.

\section{Experimental Procedures}

\subsection{Sample fabrication and microstructural analyses}

Grain sizes of nanocrystalline electrodeposited Ni-W alloys were controlled by changing the bath temperature during electrodeposition, and were determined by applying the Scherrer equation to the XRD (111) peak broadening. 


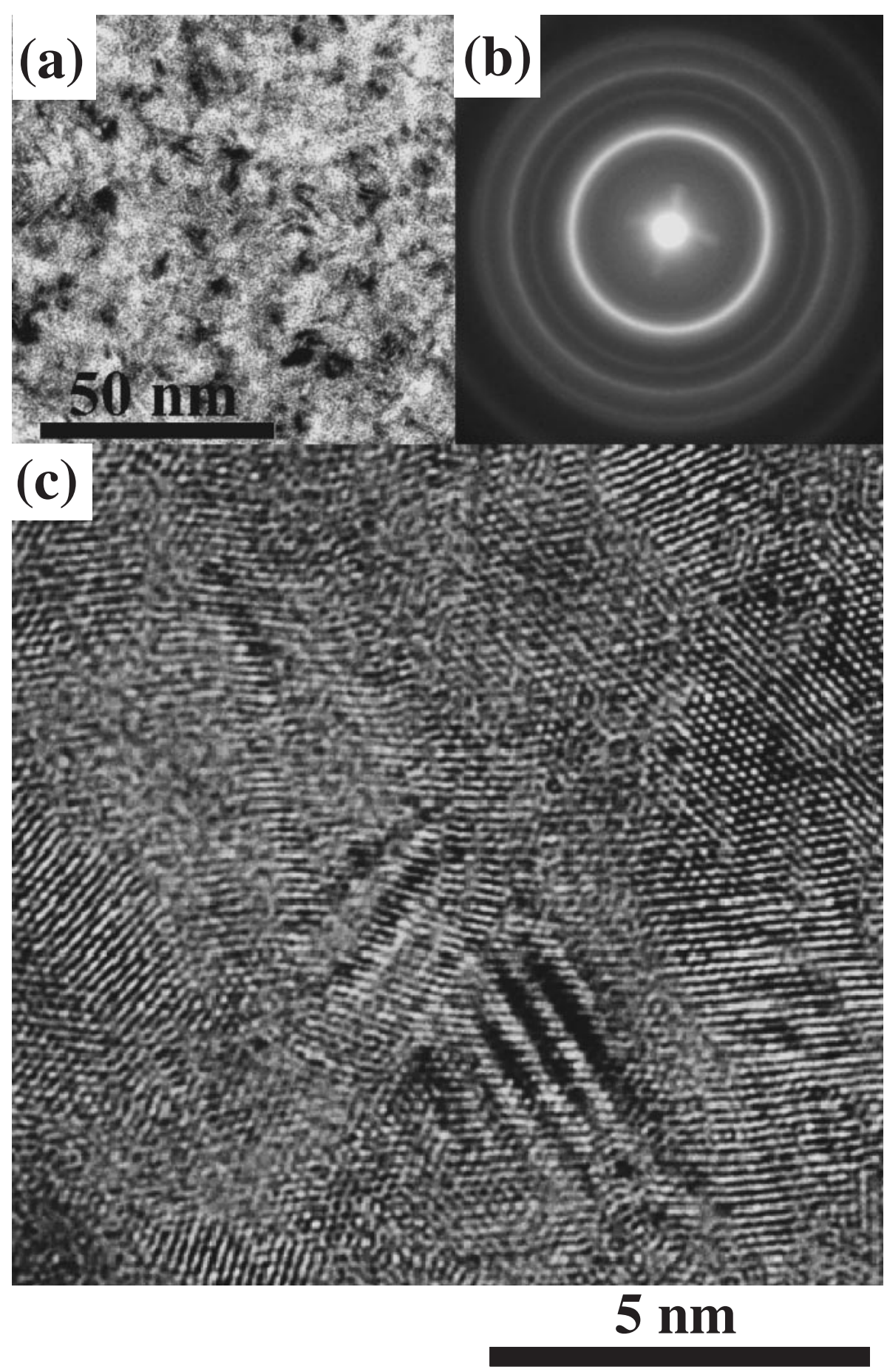

Fig. 1 Microstructure of the electrodeposited Ni-W alloy with the grain size of $5 \mathrm{~nm}$ characterized through (a) the BF image, (b) the SAED pattern and (c) the HR-TEM image.

Other process parameters in the Ni-W electrodeposition can be referred in ref. 5). The electrodeposited Ni-W alloys with grain sizes of 5 and $8 \mathrm{~nm}$ were examined through detailed characterizations.

Mirostructural observations were carried out by field emission transmission electron microscopy (FE-TEM) operated at $200 \mathrm{kV}$ on the normal planes of the electrodeposited foils. Figure 1(a) and (b) shows the typical bright-field (BF) image and selected area electron diffraction (SAED) pattern of the electrodeposited Ni-W alloy with grain size of $5 \mathrm{~nm}$, respectively. The SAED pattern indicates that the major constituent phase is fcc $\mathrm{Ni}$ which are randomly oriented. The first $\{111\}$ ring pattern has a feature of halo, suggesting the presence of an amorphous phase. Figure 1(c) shows the highresolution transmission electron microscopy (HR-TEM) image of the alloy. Disordered regions are found between the regions where lattice fringes can be clearly seen. Similar features were observed from the alloy with grain size of $8 \mathrm{~nm}$ (data not shown).

To identify the nano-phases, nano beam diffraction (NBD) analyses were carried out for more than 15 spots randomly sampled in each grain-size specimen by using the electron 


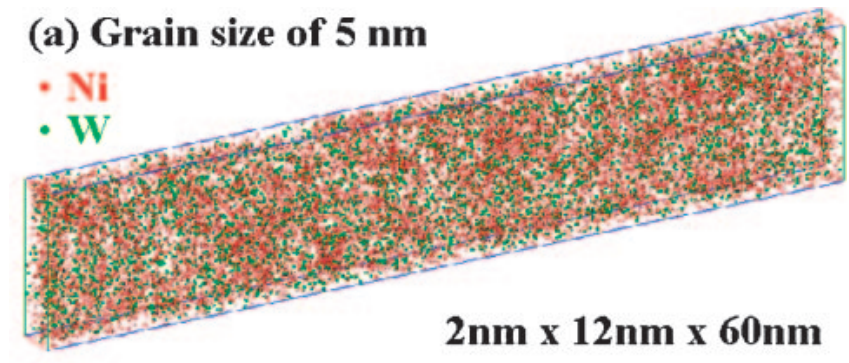

(b) Grain size of $8 \mathbf{~ n m}$

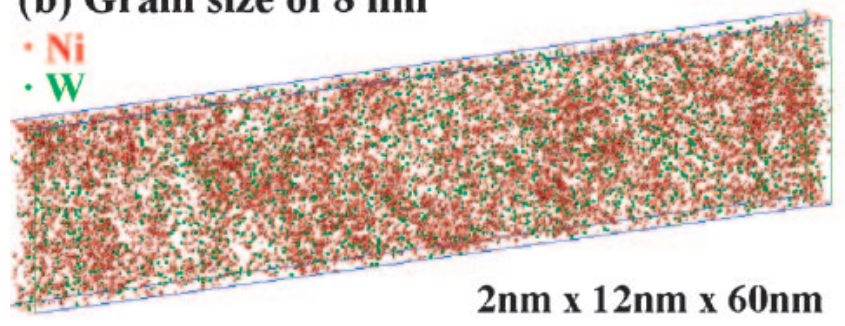

Fig. 2 3DAP atom maps of the Ni-W alloys with the grain sizes of (a) $5 \mathrm{~nm}$ and (b) $8 \mathrm{~nm}$.

beam converged to about $0.5 \mathrm{~nm}$ in diameter. Energy dispersive $\mathrm{x}$-ray spectroscopy (EDS) analyses were also performed for the corresponding spots.

\subsection{DAP analyses}

The characteristic length-scales of the nanocrystals are smaller than the thickness of the TEM specimen, and multiple phases are piled up in the thickness direction. Therefore, TEM images cannot be employed to identify the nano-phases and to evaluate the $\mathrm{W}$ concentration and the volume fraction of each phase accurately. However, 3DAP analyses are free from this limitation.

The details on the preparation of the 3DAP specimen were published elsewhere. ${ }^{21)}$ The three-dimensional atom probe, equipped with the CAMECA tomographic atom probe detection system, was used. The atom probe analyses were operated at the load voltage between $6 \mathrm{kV}$ and $10 \mathrm{kV}$ with a pulse fraction of 0.20 . A pulse repetition rate was $600 \mathrm{~Hz}$. Specimens were held at a temperature between $60 \mathrm{~K}$ and $70 \mathrm{~K}$ under an ultrahigh vacuum condition $\left(\sim 1 \times 10^{-8} \mathrm{~Pa}\right)$.

Obtained 3DAP data is the three-dimensional coordinates and element of the atoms in the analysis volume ${ }^{22}$ of approximately $12 \mathrm{~nm} \times 12 \mathrm{~nm} \times 60 \mathrm{~nm}$. Local W concentrations were computationally calculated by counting $\mathrm{Ni}$ and $\mathrm{W}$ atoms in each small cubic block in the analyzed volume (each cubic block containing averagely 50 detected atoms). A frequency distribution diagram was built for the statistical analyses of the local $\mathrm{W}$ concentrations over the all blocks in the analyzed volume.

\section{Results and Discussions}

Figure 2(a) and (b) show 3DAP atom maps (sliced to be $2 \mathrm{~nm} \times 12 \mathrm{~nm} \times 60 \mathrm{~nm}$ from the analyzed volume) of the Ni$\mathrm{W}$ alloys with the grain sizes of $5 \mathrm{~nm}$ and $8 \mathrm{~nm}$, respectively. In both figures, W-enriched and $\mathrm{W}$-depleted zones are recognized in a few nm scale dimension.

Figure 3(a) and (b) show the concentration frequency distribution of $\mathrm{W}$ atoms for the electrodeposited Ni-W alloys
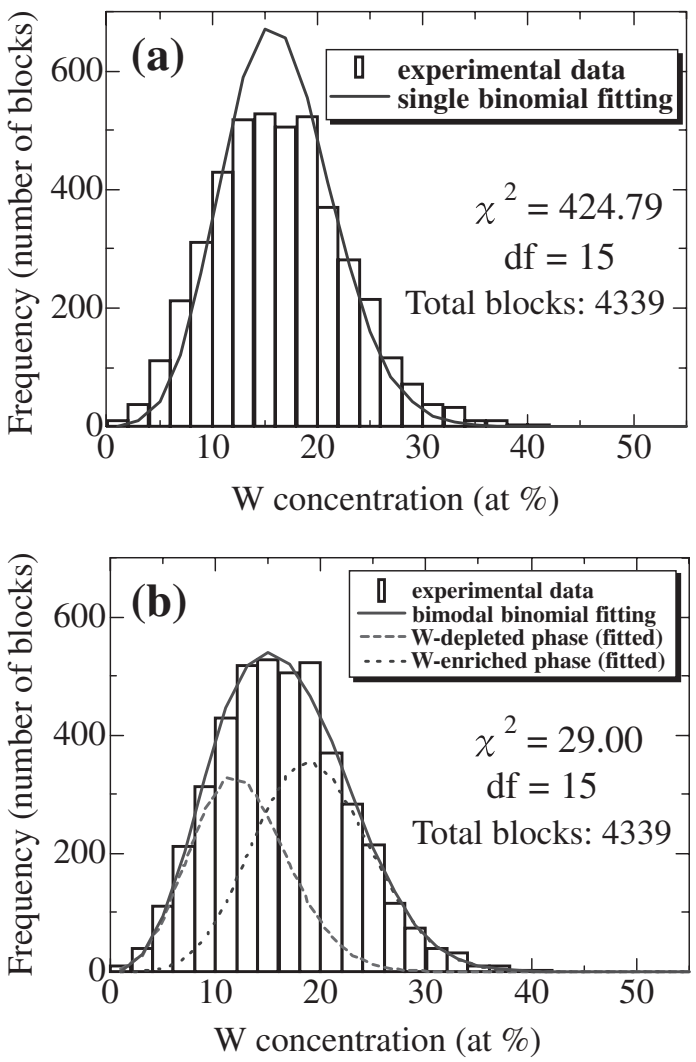

Fig. 3 Frequency distribution diagram of electrodeposited Ni-W alloy with the grain size of $5 \mathrm{~nm}$ together with (a) single binomial fitting curve and (b) bimodal binomial fitting curves. Binomial fitting curve, chi-squared value $\chi^{2}$ and degree of freedom df are also shown.

with the grain size of $5 \mathrm{~nm}$, together with the single binomial fitting curve and bimodal binomial fitting curves, respectively. If the nanostructure is composed of a single phase and the solute atoms distribute randomly, the frequency distribution should follow the single binomial distribution. To confirm that, chi-square tests were performed between the observed and fitted (single binomial) distributions in Fig. 3(a). Taking the degrees of freedom as $15, \chi^{2}$ values were calculated to be 424.79 and 470.46 for the alloys with the grain sizes of 5 and $8 \mathrm{~nm}$, respectively, which were much larger than the value of the chi-square percent point function (25.00) with a significance level of $5 \%$. From the results of the chi-squared tests, the assumption of the single phase is rejected. On the other hand, in the case of bimodal binomial fitting, the $\chi^{2}$ values were calculated to be 25.23 and 10.23 for the alloys with the grain sizes of 5 and $8 \mathrm{~nm}$, respectively, which were comparable to or smaller than 25.00. So the assumption of two phases is justified.

In the bimodal binomial fitting in Fig. 3(b), we employed the following function:

$$
f(x)=\theta b\left(x ; n, p_{1}\right)+(1-\theta) b\left(x ; n, p_{2}\right),
$$

where,

$$
b(x ; n, p)=\left(\begin{array}{l}
n \\
x
\end{array}\right) p^{x}(1-p)^{n-x},\left(\begin{array}{l}
n \\
x
\end{array}\right)=\frac{n !}{x !(n-x) !} .
$$

The average $\mathrm{W}$ concentration of the $\mathrm{W}$-depleted phase $p_{1}$ and that of the $\mathrm{W}$-enriched phase $p_{2}$ and the volume fraction of 
Table 1 Quantitative data analyzed by 3DAP and other methods for electrodeposited Ni-W alloys.

\begin{tabular}{|c|c|c|c|c|c|c|c|c|c|c|c|}
\hline \multirow{2}{*}{$\begin{array}{c}\text { Sample } \\
\text { No. }\end{array}$} & \multicolumn{2}{|c|}{ i) Overall W conc. (at\%) } & \multicolumn{3}{|c|}{ ii) Local W conc. (at\%) } & \multicolumn{3}{|c|}{ iii) Volume fraction $(\%)$} & \multicolumn{2}{|c|}{ iv) Grain size (nm) } & \multirow{2}{*}{ Ref. } \\
\hline & value(s) & method(s) & cry.* & amo.* & method & cry.* & amo.* & method & value(s) & method(s) & \\
\hline$\# 1$ & \multirow{2}{*}{18} & \multirow{2}{*}{ unknown } & $12 \pm 1$ & $32 \pm 2$ & \multirow{2}{*}{ ii)-a } & - & - & - & $4 \pm 1$ & \multirow{2}{*}{ iv)-a } & \multirow{2}{*}{ 19) } \\
\hline$\# 2$ & & & $6 \pm 1$ & $38 \pm 3$ & & - & - & - & $3 \pm 1$ & & \\
\hline \#3 & $17.5 \pm 1.5$ & \multirow{3}{*}{ i)-b } & 14.0 & 20.1 & \multirow{3}{*}{ ii)-b } & 42.2 & 57.8 & & $3 / 2$ & \multirow{3}{*}{ iv)-b } & \multirow{3}{*}{ 20) } \\
\hline$\# 4$ & $14.5 \pm 1.4$ & & 13.1 & 19.8 & & 79.1 & 20.9 & iii)-b & $9 / 9$ & & \\
\hline$\# 5$ & $10.7 \pm 1.2$ & & 9.9 & 17.3 & & 89.2 & 10.8 & & $17 / 17$ & & \\
\hline$\# 6$ & $17.7 / 18.3$ & \multirow{2}{*}{ i)-c } & 10.9 & 17.9 & \multirow{2}{*}{ ii)-b } & 36.6 & 63.4 & \multirow{2}{*}{ iii)-c } & 5.0 & \multirow{2}{*}{ iv)-c } & This \\
\hline$\# 7$ & $16.7 / 17.2$ & & 13.7 & 20.5 & & 51.0 & 49.0 & & 8.0 & & work \\
\hline
\end{tabular}

i)-b: directly from the number of detected $\mathrm{Ni}$ and $\mathrm{W}$ atoms in the 3DAP analyzed volume.

i)-c: directly from the number of detected $\mathrm{Ni}$ and $\mathrm{W}$ atoms in the 3DAP analyzed volume. / from inductively coupled plasma mass spectrometry (ICPMS)

ii)-a: from ladder diagrams obtained in 3DAP analyses (The transitional local W concentration is not given)

ii)-b: from the fitting parameters in bimodal binomial distribution function to the 3DAP frequency distribution diagram.

iii)-b: from the analytical expression for the volume fraction of the crystalline phase $f_{\text {cry }}=(d-t)^{3} / d^{3}$ (the grain-boundary thickness $t=0.75$ nm).

iii)-c: from the fitting parameter in bimodal binomial distribution function to the 3DAP frequency distribution diagram.

iv)-a: from ladder diagrams obtained in 3DAP analyses. (The transitional local $\mathrm{W}$ concentration is not given.)

iv)-b: from autocorrelation function analyses of the spatial $\mathrm{W}$ concentration distribution in 3DAP analyzed volume. / by applying the Sherrer equation to the (111) peak broadening.

iv)-c: by applying the Sherrer equation to the (111) peak broadening.

*The amorphous phase had been referred to as grain boundary phase by the past researchers. ${ }^{19,20)}$

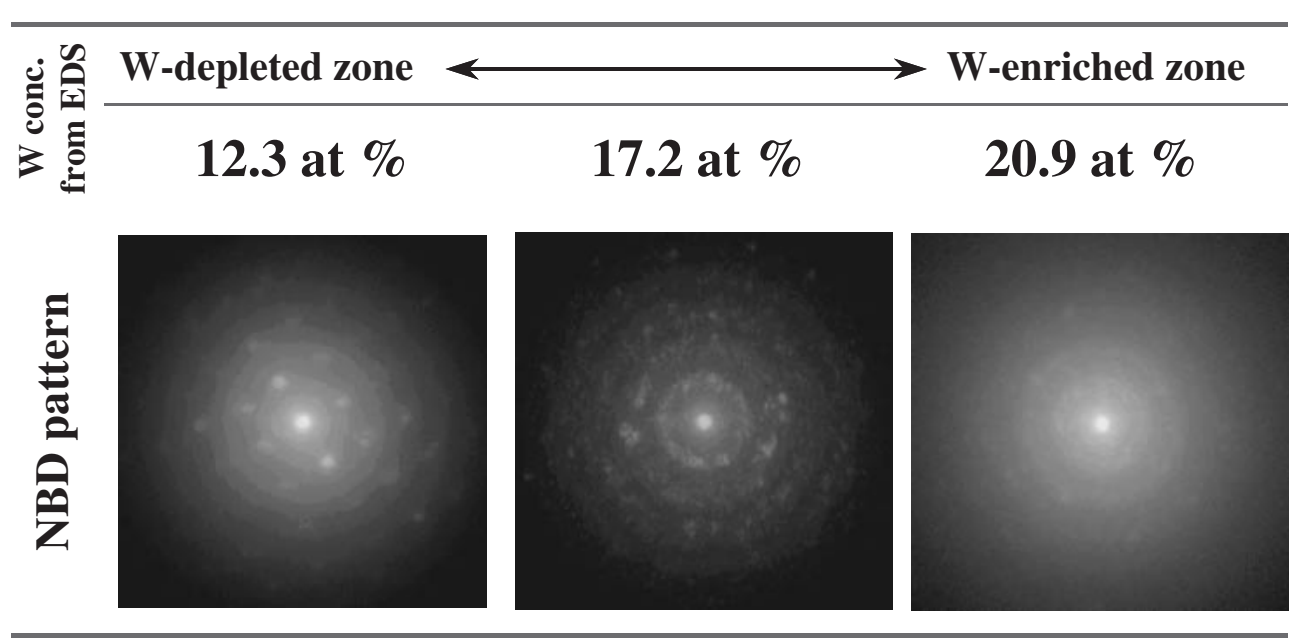

Fig. 4 Typical NBD patterns of the electrodeposited Ni-W alloys with various W concentrations evaluated from EDS analyses.

the W-depleted phase $\theta$ were optimized to fit the experimental data. The volume fractions, the average $\mathrm{W}$ concentrations and the grain sizes are summarized in Table 1 together with the data from literatures. ${ }^{19,20)}$ The average $\mathrm{W}$ concentrations of the $\mathrm{W}$-depleted and $\mathrm{W}$-enriched phases are 10.9 at $\%$ and 17.9 at $\%$, respectively, for the sample with the grain size of $5 \mathrm{~nm}$. In the case of the grain size of $8 \mathrm{~nm}$, those of the $\mathrm{W}$-depleted and $\mathrm{W}$-enriched phases are 13.7 at $\%$ and 20.5 at $\%$, respectively. Both of the histograms are composed of two peaks at around 10 at $\%$ and 20 at $\%$.

The observed NBD patterns of the alloy with the grain size of $5 \mathrm{~nm}$ are shown in Fig. 4. In the NBD analyses, diffraction spots are necessarily seen when the sample contains a periodic structure with the length of more than $1 \mathrm{~nm}$. Among the NBD patterns for the electrodeposited Ni-W alloys with the grain sizes of 5 and $8 \mathrm{~nm}$, there were quite a few patterns without any diffraction spots. Therefore, it leaves no doubt that the amorphous phase exists in the alloys. The W concentrations at the corresponding spot evaluated from EDS analyses are also shown in Fig. 4. As the W concentration increases, the NBD patterns transit from Debye rings indicating a crystalline phase to the halo patterns indicating an amorphous phase. It is noted that the $\mathrm{W}$ concentrations leading the Debye rings and halo patterns are corresponding to in the range of the $\mathrm{W}$-depleted phase (around 10 at\%) and $\mathrm{W}$-enriched phases (around 20 at\%) in Fig. 3, respectively. Although the NBD patterns do not exhibit very good contrast because of the overlapping phases, the similar trends are seen in the results of NBD and EDS analyses for the alloy with the grain size of $8 \mathrm{~nm}$. Based on these results, W-depleted and $\mathrm{W}$-enriched phases are identified as nanocrystalline and amorphous phases, respectively.

Figure 5(a) and (b) show the visualizations of the Wconcentration distribution on the cross section of the alloys with grain size of 5 and $8 \mathrm{~nm}$, respectively. It can be seen that the darker area is more widely distributed in Fig. 5(b) than in Fig. 5(a). It is consistent with the data for the samples \#6 and \#7 in Table 1, showing that the volume fraction of crystalline 


\section{(a) Grain size of $5 \mathbf{~ n m}$}

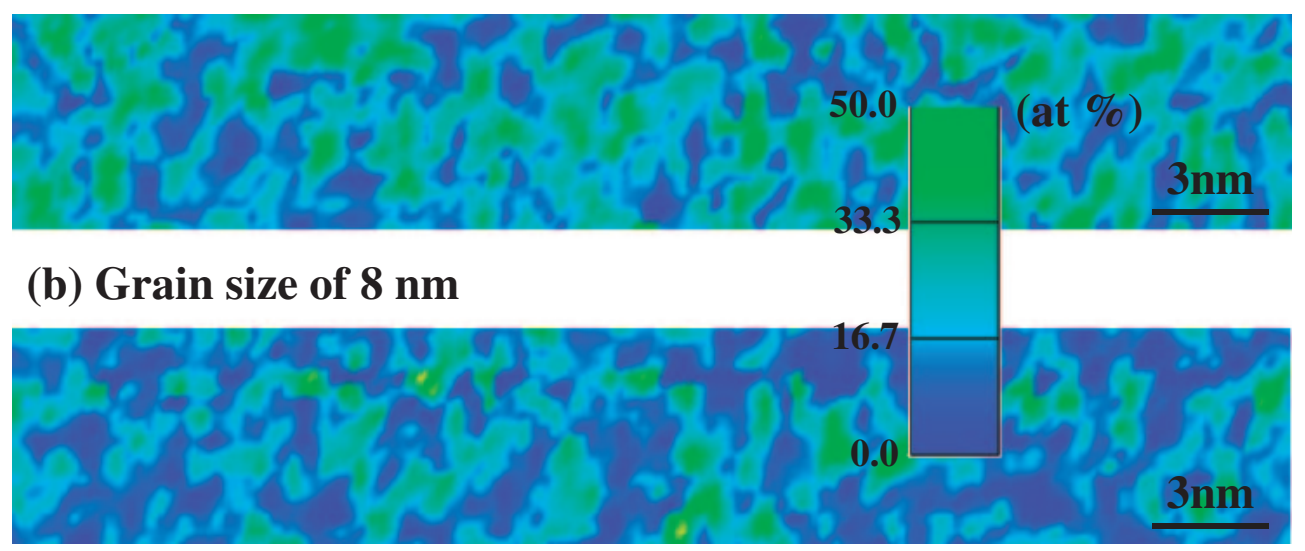

Fig. 5 W concentration visualizations on cross section of the alloys with grain size of (a) $5 \mathrm{~nm}$ and (b) $8 \mathrm{~nm}$. The regions with low W concentration are painted in dark color (blue), while high W concentration in brighter color (light green).

phase in the alloy with the grain size of $8 \mathrm{~nm}$ is large compared to that in the alloy with the grain size of $5 \mathrm{~nm}$. It can be seen from the visualization in Fig. 5(a) and 5(b) that these alloys are duplex composites, rather than the nanocrystals embedded in the amorphous matrix.

These findings obtained by 3DAP and NBD analyses have thoroughly demonstrated that the electrodeposited Ni-W alloys with grain size of 5 and $8 \mathrm{~nm}$ are actually the duplex nanocomposite alloys containing W-enriched amorphous phase and $\mathrm{W}$-depleted nanocrystalline phase.

\section{Conclusion}

Electrodeposited Ni-W alloys with grain size of 5 and $8 \mathrm{~nm}$ were successfully characterized through a series of nanocharacterization techniques. The W-concentration histograms obtained from 3DAP data fitted well to the bimodal binomial distributions, and demonstrated the two-phase coexistence in the alloys. Compiling the results of NBD analyses and $\mathrm{W}$-concentration histograms, the $\mathrm{W}$-depleted and $\mathrm{W}$-enriched phases are identified as nanocrystalline and amorphous phases, respectively. The electrodeposited Ni-W alloys with the grain sizes of 5 and $8 \mathrm{~nm}$ have been qualitatively and quantitatively proved to be the amorphous/nanocrystalline duplex composites.

\section{Acknowledgement}

This work was in part supported by the Grant in Aid of the Ministry of Education, Sports, Culture, Science and Technology, Priority Area 'Materials Science of Bulk Metallic Glasses'.

\section{REFERENCES}

1) K. S. Siow, A. A. O. Tay and P. Oruganti: Mater. Sci. Technol. 20 (2004) 285-294.

2) A. Inoue and A. Takeuchi: Mater. Trans. 43 (2002) 1892-1906.

3) K. Lu: Mater. Sci. Eng. R 16 (1996) 161-221.

4) A. H. Chokshi, A. Rosen, J. Karch and H. Gleiter: Scripta Metall. 23 (1989) 1679-1684.

5) A. Giga, Y. Kimoto, Y. Takigawa and K. Higashi: Scripta Mater. 55 (2006) 143-146.

6) G. Herzer: IEEE Trans. Magn. 26 (1990) 1397-1402.

7) C. T. J. Low, R. G. A. Wills and F. C. Walsh: Surf. Coat. Technol. 201 (2006) 371-383.

8) Y. L. Chiu, N. Baluc and R. Schäublin: Int. J. Mod. Phys. 20 (2006) 4195-4200.

9) N. Sulitanu and F. Brînzã: Mater. Sci. Eng. B 106 (2004) 155-162.

10) N. Sulitanu: J. Magn. Magn. Mater. 231 (2001) 85-93.

11) K. Itoh, F. Wang and T. Watanabe: J. Jpn. Inst. Met. 65 (2001) 10231028.

12) L. Zhu, O. Younes, N. Achkenasy, Y. Shacham-Diamand and E. Gileadi: Appl. Surf. Sci. 200 (2002) 1-14.

13) T. Yamasaki, P. Schlossmacher, K. Ehrlich and Y. Ogino: Nanostruct. Mater. 10 (1998) 375-388.

14) H. S. Kim: Scripta Mater. 39 (1998) 1057-1061.

15) H. Somekawa, T. G. Nieh and K. Higashi: Scripta Mater. 50 (2004) 1361-1365.

16) D. Nagahama, T. Ohkubo and K. Hono: Scripta Mater. 49 (2003) 729734.

17) T. Honma, S. Yanagita, K. Hono, Y. Nagai and M. Hasegawa: Acta Mater. 52 (2004) 1997-2003.

18) S. Ohsaki, K. Hono, H. Hidaka and S. Takagi: Scripta Mater. 52 (2005) 271-276.

19) P. Choi, T. Al-Kassab, F. Gärtner, H. Kreye and R. Kirchheim: Mater. Sci. Eng. A 353 (2003) 74-79.

20) A. J. Detor, M. K. Miller and C. A. Schuh: Philos. Mag. 86 (2006) 4459-4475.

21) K. Hono: Prog. Mater. Sci. 47 (2002) 621-729.

22) K. Hono: Acta Mater. 47 (1999) 3127-3145. 